\title{
Perfluoroarene-based peptide macrocycles that inhibit the Nrf2/Keap1 interaction.
}

\author{
Richard J. Steel $^{\mathrm{a}}$, Maria A. O’Connell ${ }^{\mathrm{a}, *}$ and Mark Searcey ${ }^{\mathrm{a}, \mathrm{b}} *$ \\ ${ }^{a}$ School of Pharmacy, University of East Anglia, Norwich Research Park, Norwich, UK, NR4 7TJ \\ ${ }^{b}$ School of Chemistry, University of East Anglia, Norwich Research Park, Norwich, UK, NR4 7TJ
}

\begin{abstract}
ARTICLE INFO
ABSTRACT

Article history:

Received

Revised

Accepted

Available online

Keywords:

The Nrf2/Keap1 interaction is a target in the development of new therapeutic agents, where inhibition of the interaction activates Nrf2 and leads to the generation of downstream antiinflammatory effects. Peptides that mimic the B-turn in the Keap1 active site and are constrained by a disulfide bridge have high affinity for Keap1 but no intracellular activity. The introduction of a perfluoroalkyl- bridging group to constrain the peptides, coupled with glutamic acid to proline replacement leads to a new peptide with a $K_{i}$ of $6.1 \mathrm{nM}$ for the Nrf2/Keap1 binding interaction, although this does not translate into intracellular activity.
\end{abstract}

Nrf2

Keap1

Protein-protein interaction

Hexafluorobenzene

Peptide
Due to its central role in several processes associated with inflammation, ${ }^{1}$ the activation of Nrf2 (Nuclear factor (erythroidderived 2)-like 2) has become an attractive target for the development of therapeutic interventions. ${ }^{2}$ As a transcription factor, Nrf2 is involved in the activation of various pathways that are associated with cellular stress. The binding of Nrf2 to DNA leads to the production of proteins such as heme-oxygenase 1 (HO$1)^{3}$ and NAD(P)H:quinone oxidoreductase $1(\mathrm{NQO} 1)^{4}$ that play a protective role in the cell. Nrf2 is closely controlled by the presence of the protein Kelch-like ECH-associated protein 1 (Keap1), which binds to it and induces ubiquitination and proteasome mediated degradation. ${ }^{5}$ This then leads to the observation that the inhibition of the Nrf2/Keap1 interaction, a protein-protein interaction (PPI), could lead to the activation of Nrf2, which could have a subsequent beneficial effect in acute and chronic conditions involving inflammation.

In the clinic, Nrf2 activation is involved in the use of dimethyl fumarate to treat multiple sclerosis ${ }^{6}$ and clinical evaluation of bardoxolone in the treatment of chronic kidney disease is still continuing. ${ }^{7}$ Both of these compounds are likely to interact in a covalent fashion with Keap1.

Efforts to target the Nrf2/Keap1 interaction that do not involve covalent modification have begun to gather pace over the last few years. The interaction involves two binding sites on Keap1 - a high affinity ETGE sequence and a low affinity DLG site. ${ }^{8}$ The ETGE site involves a loop from Nrf2 and studies by Hannink and coworkers $^{9}$ showed that a 14 mer sequence LQLDEETGEFLPIQ could bind to the site with high affinity. We later showed that conjugation of this sequence to a cell penetrating TAT-peptide led to a molecule with the ability to activate an anti-inflammatory response in THP1 human monocytic cells. ${ }^{10}$ As well as other peptide based approaches, ${ }^{11-14}$ there have been several small molecules developed to target the Nrf2/Keap1 interaction, based upon library screening, structure based ligand design or click chemistry. ${ }^{15-18}$

As the peptide that binds to the ETGE sequence forms a loop or turn structure (Fig 1A), one intriguing approach to generating high affinity peptidic structures is to generate cyclic peptides. ${ }^{19}$ Such structures can often have advantages of stability, affinity and cellular uptake over linear peptides. One of the simplest approaches to generating a cyclic peptide is to generate a disulfide bridge and indeed, a recent publication has demonstrated that cyclic peptides based upon the Nrf2 binding motif have a high affinity for Keap $1 .{ }^{20}$ This has prompted us to disclose our own investigations of cyclic peptides targeting the Nrf2/Keap1 interaction. Herein, we show that our disulfide bridged compounds had high binding affinity to Keap1 compared with the linear structures but were inactive in cells. Incorporation of a staple through reaction with hexafluorobenzene generated the first perfluoroarene stapled peptides ${ }^{21.22}$ targeting the Nrf2/Keap1 interaction and gave a cyclic peptide that was amongst the highest affinity binding peptides that we have studied, however stapling in this way did not generate intracellularly active molecules. 


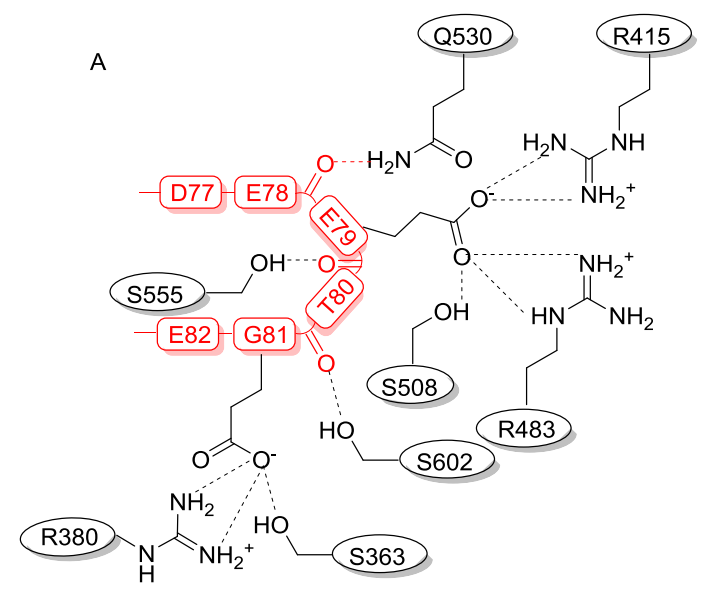

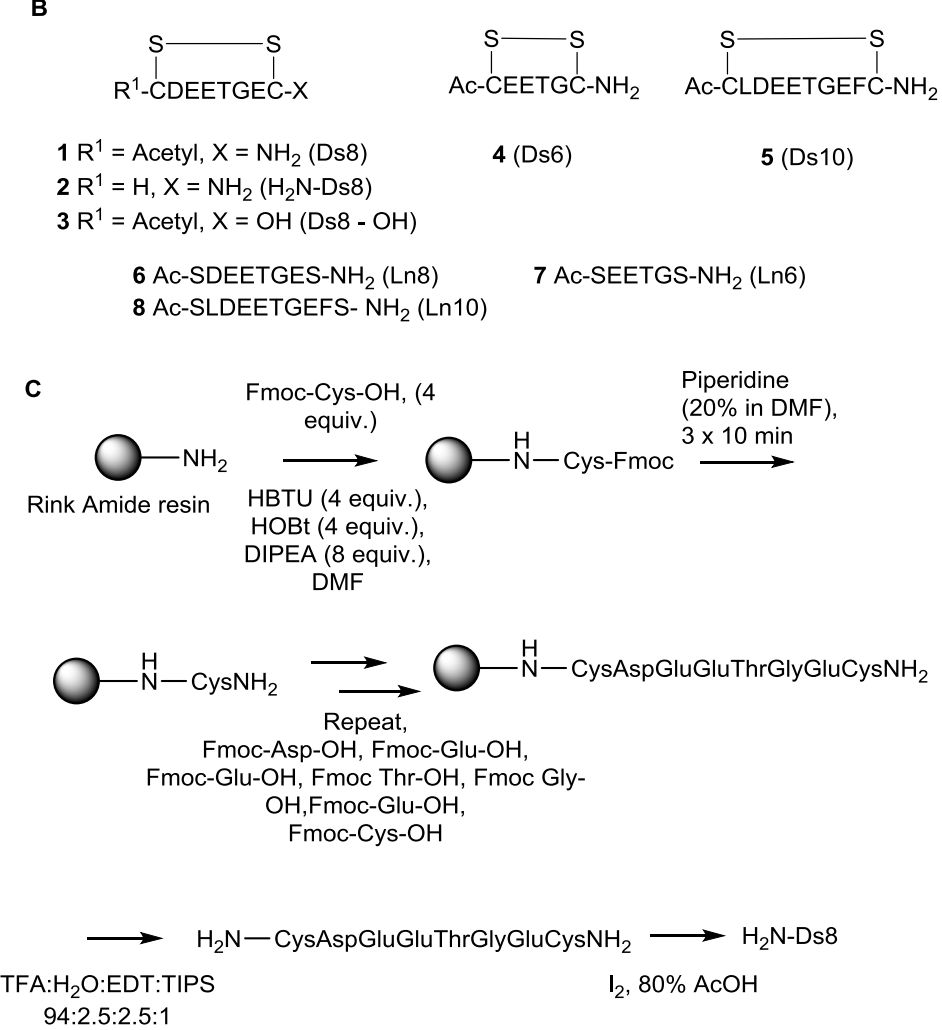

$1 \mathrm{R}^{1}=$ Acetyl, $\mathrm{X}=\mathrm{NH}_{2}$ (Ds8)

$2 \mathrm{R}^{1}=\mathrm{H}, \mathrm{X}=\mathrm{NH}_{2}\left(\mathrm{H}_{2} \mathrm{~N}-\mathrm{Ds} 8\right)$

$3 \mathrm{R}^{1}=$ Acetyl, $\mathrm{X}=\mathrm{OH}(\mathrm{Ds} 8-\mathrm{OH})$

6 Ac-SDEETGES-NH $\mathrm{N}_{2}(\mathrm{Ln} 8)$ 8 Ac-SLDEETGEFS- $\mathrm{NH}_{2}(\mathrm{Ln} 10)$

Fmoc-Asp-OH, Fmoc-Glu-OH,

Fmoc-Glu-OH, Fmoc Thr-OH, Fmoc Gly-

$\mathrm{OH}, \mathrm{Fmoc}-\mathrm{Glu}-\mathrm{OH}$

Fmoc-Cys-OH

4 (Ds6) 5 (Ds10)

7 Ac-SEETGS-NH $2(\operatorname{Ln} 6)$

(1)

(n)

Figure 1 A. Interactions between the Nrf2 DEETGE (in red) and the Keap1 binding site, showing the turn structure adopted by the Nrf2 in the binding site. B. The peptide sequences used for the first part of this study. C. Example of the route to synthesis of the peptides, in this case $\mathrm{H}_{2} \mathrm{~N}-\mathrm{Ds} 8$ (2) See supporting information for a full description. HBTU = O-benzotriazole- $N, N, N^{\prime}, N$ '-tetramethyl-uronium-hexafluoro-phosphate, HOBt $=N$-Hydroxybenzotriazole, DIPEA = $N, N$-diisopropylethylamine, $\mathrm{DMF}=N, N$-Dimethylformamide, TFA = Trifluoroacetic acid, EDT = 1,2-ethanedithiol, TIPS $=$ Triisopropylsilane.

In order to study the effects of cyclising the peptide through a disulfide bridge, we chose three sequences to flank with cysteines - the core DEETGE motif (CDEETGEC, Ds8), a longer motif with the amino acids generating LDEETGEF (CLDEETGEFC, Ds10) and a truncated version containing only the central ETGE (CETGEC, Ds6) (Fig 1B). The linear sequences were generated on Rink amide resin ${ }^{23}$ to give the $C$-terminal amide compounds and were acetylated on the $N$-terminus. To assess the appropriate nature of these end groups, Ds8 was also made without the $\mathrm{N}$ terminal acetylation $\left(\mathrm{H}_{2} \mathrm{~N}\right.$-Ds8) (Fig $1 \mathrm{C}$ ) and on 2-chlorotrityl resin to generate the free acid at the $C$-terminus (Ds8-OH). Oxidation of the peptides with $\mathrm{I}_{2} / \mathrm{AcOH}$ formed the cyclic peptides. All compounds were purified by HPLC and their identities were confirmed by MALDI-TOF MS (See Supporting Information for full details).

The ability of the peptides to inhibit the Nrf2/Keap1 interaction was initially assessed by a fluorescence polarisation assay. ${ }^{24}$ The 14mer peptide LQLDEETGEFLPIQ was labelled at the $N$ terminus via reaction with fluorescein isothiocyanate in DMSO with triethylamine as base. The product (F-14) was purified by preparative HPLC and identified through MALDI-TOF mass spectrometry. The assay was optimised to utilise the Keap1 Kelch domain (352-609) at a $30 \mathrm{nM}$ final concentration and $5 \mathrm{nM} \mathrm{F-14.}$ A sample inhibition curve (for Ds8) in shown in Figure 2 and the resulting values for the disulfide peptides in Table 1.

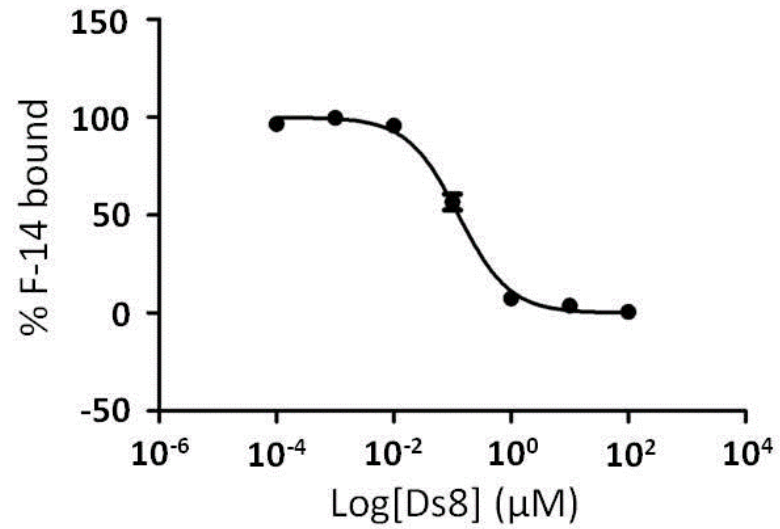

Figure 2. FP inhibition by Ds8 peptide. $\mathrm{n}$ = 3, 30 nM Keap1, 5 nM F14 


\begin{tabular}{|c|c|c|c|c|}
\hline & Peptide & $\mathrm{K}_{\mathrm{i}}(\mathrm{nM})$ & $95 \% \mathrm{Cl}(\mathrm{nM})$ & $\mathrm{R}^{2}$ \\
\hline $\mathbf{1}$ & Ds8 & 95 & $73.2-123.3$ & 0.9899 \\
\hline $\mathbf{6}$ & Ln8 & 1300 & $1100-1500$ & 0.9942 \\
\hline $\mathbf{4}$ & Ds6 & 151500 & $88800-$ & 0.9522 \\
& & & 258700 & \\
\hline $\mathbf{7}$ & Ln6 & 280400 & $116200-$ & 0.9246 \\
& & & 676200 & \\
\hline $\mathbf{5}$ & Ds10 & 47.9 & $33.1-69.2$ & 0.9807 \\
\hline $\mathbf{8}$ & Ln10 & 176.9 & $135.6-230.6$ & 0.9900 \\
\hline $\mathbf{3}$ & Ds8-OH & 47.7 & $35.5-64.1$ & 0.9875 \\
\hline $\mathbf{2}$ & $\mathrm{H}_{2} \mathrm{~N}-\mathrm{Ds} 8$ & 274.9 & $182.6-413.8$ & 0.9770 \\
\hline
\end{tabular}

of a $\beta$-turn, while the glutamic acid replaced (equivalent to Glu78 in the human Nrf2 sequence, see Fig 1A) does not make a significant contribution to binding interactions. As such, we also synthesised the peptides CDPETGEC (Ar8P, 11) and CLDPETGEFC (Ar10P, 12) equivalent to Ln8 and Ln10 respectively. When subjected to reaction with hexafluorobenzene under similar conditions, these two compounds generated the perfluoroaryl peptide macrocycles Ar8P and Ar10P.

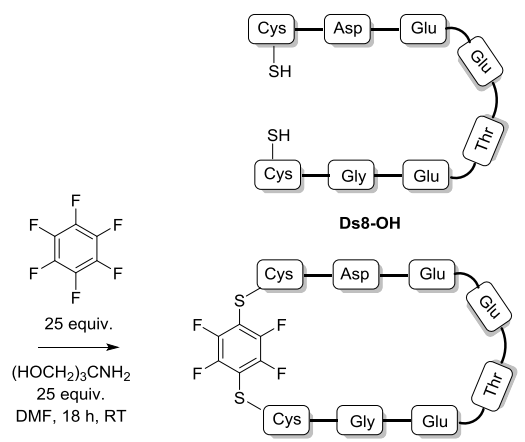

Table 1. $\mathrm{K}_{\mathrm{i}}$ values for Fp inhibition by cyclic disulfides, $\mathrm{n}=$ 3, 30 nM Keap1, 5 nM F-14. CI - confidence intervals. For calculations see supporting information. $\mathrm{Ln}=$ linear

Clearly, cyclisation of the Ds8 peptide had a significant effect on the binding affinity - with $>10$ fold increase in $\mathrm{K}_{\mathrm{i}}$ for Ds8 over the linear peptide and over 25 fold for Ds8-OH. Ds10 also shows a modest increase over the linear peptide, maintaining low $\mathrm{nM}$ activity. It is also clear that the Ds6 peptide is too short to maintain good binding affinity.

These compounds were then assessed for the ability to activate HO-1 expression in THP1 cells using RT-PCR and in comparison with TAT14. None of the compounds exerted a biological effect at any of the time points investigated. This is strongly suggestive that, even in the cyclic form, these compounds are either not entering the cells, or are being reduced to a linear form and metabolised during uptake.

"Peptide stapling" involves the constraining of peptides in particular conformations through the incorporation of novel linker groups. Most of the focus of this approach has been on the introduction of groups that stabilise an $\alpha$-helix in systems targeting, for example, the p53/MDM2 protein-protein interaction. ${ }^{25}$ Olefin metathesis reactions have been popular in this context and there has been some discussion as to whether groups that are incorporated into a peptide also facilitate cellular uptake. ${ }^{26}$ One of the drawbacks of many of these methods is that they involve the incorporation of non-natural amino acids, sometimes generated through multistep synthetic routes, so that more synthetically tractable and simple approaches are highly desirable.

One such simple approach was recently described by Pentelute and co-workers ${ }^{21}$ and involves macrocyclisation through the reaction of cysteines with hexafluorobenzene or decafluorobiphenyl under mild conditions. This chemistry was successfully applied to the generation of relatively stable $\alpha$-helical Her-2-affibodies and was also shown to increase the penetration of peptides through the blood-brain barrier. ${ }^{22}$ As such, this represented a potential approach to generate constrained $\beta$-turn type cyclic peptides for the Nrf2/Keap1 interaction under mild conditions.

In order to generate the perfluoroaryl peptide macrocycles, Ln6, Ln8 and Ln10 were resynthesised, cleaved from the resin and purified. They were then treated with a large excess (25 equivalents) of a solution of hexafluorobenzene (50 mM) and TRIS-base $(25 \mathrm{mM})$ in DMF at room temperature for $18 \mathrm{~h}$ (Scheme 1). Following purification both Ln6 and Ln8 starting materials generated the target compounds Ar6 (9) and Ar8 (10), whereas, somewhat surprisingly, the Ln10 only gave the disulfide bridged analogue Ds10. Two further peptides were also synthesised. Wells and co-workers ${ }^{12}$ have previously shown that the incorporation of a proline in place of glutamic acid in the sequence DEETGE (i.e. DPETGE) helps to induce the formation

\begin{tabular}{|c|c|c|c|c|}
\hline & Peptide & $\mathrm{K}_{\mathrm{i}}(\mathrm{nM})$ & $95 \% \mathrm{Cl}(\mathrm{nM})$ & $\mathrm{R}^{2}$ \\
\hline $\mathbf{9}$ & Ar6 & - & - & - \\
\hline $\mathbf{1 0}$ & Ar8 & 1400 & $1100-1700$ & 0.9914 \\
\hline $\mathbf{1 1}$ & Ar8P & 6.1 & $4.4-8,5$ & 0.9827 \\
\hline $\mathbf{1 2}$ & Ar10P & 61.9 & $46.1-83.0$ & 0.9861 \\
\hline
\end{tabular}

Table 2. $\mathrm{K}_{\mathrm{i}}$ values for $\mathrm{Fp}$ inhibition by perfluoroarene compounds, $\mathrm{n}=3$, $30 \mathrm{nM}$ Keap1, $5 \mathrm{nM}$ F-14. CI - confidence intervals. For calculations see supporting information.

The Ar6 peptide showed no binding to Keap1 at concentrations that were tested in this assay. Somewhat disappointingly, the binding affinity for Ar8, in which the disulfide bridge has been crosslinked by the perfluoroaryl group, is almost 15-fold lower than the value for the disulfide compound and is more similar to the linear peptide (see Table 1). This suggests that the increased size of the cyclic peptide induced by the introduction of the aryl group means that the "fit" of the turn structure within the binding site is no longer appropriate. Introduction of the proline residue in place of a Glu in compound $\mathbf{1 1}$ (Ar8P) leads to a molecule that is amongst the most potent that we have seen in this assay, with a $\mathrm{K}_{\mathrm{i}}$ of $6.1 \mathrm{nM}$, this time 15-fold higher than Ds8. The less rigid, longer cyclic peptide 12 (Ar10P) is almost equipotent with 5.

Although it has been reported that the introduction of the perfluoroaryl linker can lead to an increase in cellular uptake, none of the compounds had measurable activity in THP-1 cells when studied by RT-PCR for their effects on HO-1 message production, or on HO-1 protein expression studied by western blot analysis, when cells were stimulated by LPS. HO-1 is a downstream product of activation of Nrf2 as a transcription factor. ${ }^{3,} 10$ This is presumably an effect of restricted access for these molecules into the cell and to their site of action. All compounds in the study were also examined for their anti-proliferative activity in THP-1 cells and no effect was observed.

In summary, peptides were designed to bind to the Keap1 protein and inhibit its interaction with Nrf2. These peptides mimicked the $\beta$-turn of the Nrf2 sequence containing DEEGTE. While constraining the peptides with a disulfide bridge gave high affinity binders and confirmed the result seen by Jiang and coworkers, ${ }^{20}$ these compounds did not have intracellular activity. As this could be because the compounds either do not penetrate the cell membrane or because they are reduced in the cellular 
environment, we incorporated a perfluoroalkyl bridge using hexafluorobenzene to both stabilise the cyclic structure and also to introduce a group that had been shown to enhance cell penetration. Both compounds Ar8P and Ar10P had a high affinity for the Keap1 binding site, with a $\mathrm{K}_{\mathrm{i}}$ for Ar8P of $6.1 \mathrm{nM}$ being the lowest value that we have observed using peptides and small molecules in an Fp assay. Unfortunately, this high affinity did not translate into cellular activity and research is continuing to generate analogues of Ar8P with similar binding affinity but enhanced cellular penetration.

Acknowlegements This paper is dedicated to our mentor and friend Professor Dale L. Boger on the occasion of his $65^{\text {th }}$ birthday. We thank the EPSRC National Mass Spectrometry Service in Swansea, UK for mass spectra.

\section{References}

1. Hayes, J. D.; Dinkova,-Kostava, A. T. Trends Biochem. Sci. 2014, 39, 199.

2. Lu, M. C.; JI, J. A.; Jiang, Z. -Y.; You, Q, D. Med. Res. Rev. 2016, 36, 924

3. Alam, J.; Stewart, D.; Touchard, C.; Boinapally, S.; Choi, A. M.; Cook, J. L. J. Biol. Chem. 1999, 274, 26071.

4. Venugopal, R.; Jaiswal, A. K. Proc. Natl. Acad. Sci. USA, 1996, 93, 14960 .

5. Itoh, K.; Wakabayahsi, N.; Katoh, Y.; Ishii, T.; Igarashi, K.; Engel, J. D.; Yamamoto, M. Genes Dev. 1999, 13, 76.

6. Suneetha, A.; Raja Rajeswari, K. J Chromatogr. B Analyt. Technol. Biomed. Life Sci. 2016, 1019, 15.

7. Wang, Y. Y.; Yang, Y. X.; Zhe, H.; Zhou, S. F. Drug Des. Devel. Ther. 2014, 8, 2075.

8. Tong, K. I.; Katoh, Y.; Kusunoki, H.; Itoh, K.; Tanaka, T.; Yamamoto, M. Mol. Cell Biol. 2006, 26, 2887.

9. Lo, S. C.; Li, X.; Henzl, M. T.; Beamer, L. J.; Hannink, M. EMBO J. 2006, 25, 3605.

10. Steel, R.; Cowan, J.; Payerne, E.; O’Connell, M. A.; Searcey, M. ACS Med. Chem. Lett. 2012, 3, 407.

11. Hancock, R.; Bertrand, H. C.; Tsujita, T.; Naz, S.; El-Bakry, A.; Laoruchupong, J.; Hayes, J. D.; Wells, G. Free Radic. Biol. Med. 2012, 52, 444.

12. Hancock, R.; Schaap, M.; Pfister, H.; Wells, G. Org. Biomol. Chem. 2013, 11, 3553.

13. Chen, Y.; Inoyama, D.; Kong, A.N.T.; Beamer, L. J.; Hu, L.; Chem. Biol. Drug. Des. 2011, 78, 1014.

14. Inoyama, D.; Chen, Y.; Huang, X.; Beamer, L.J.; Kong, A. N.; Hu, L. J. Biomol. Screen. 2012, 17, 435.

15. Jnoff, E.; Albrecht, C.; Barker, J. J.; Barker, O.; Beaumont, E.; Bromidge, S.; Brookfield, F.; Brooks, M.; Bubert, C.; Ceska, T.; Corden, V.; Dawson, G.; Duclos, S.; Fryatt, T.; Genicot, C.; Jigorel, E.; Kwong, J.; Maghames, R.; Mushi, I.; Pike, R.; Sands Z A.; Smith, M. A.; Stimson, C. C.; Courade, J. P. Chemmedchem 2014, 9, 699.

16. Hu, L.; Magesh, S.; Chen, L.; Wang, L.; Lewis, T. A.; Chen, Y.; Khodier, C.; Inoyama, D.; Beamer, L. J.; Emge, T. J.; Shen, J.; Kerrigan, J. E.; Kong, A. N.; Dandapani, S.; Palmer, M.; Schreiber, S. L.; Munoz, B. Bioorg. Med. Chem. Lett. 2013, 23 , 3039.

17. Jiang, Z. -Y.; Xu, L. L.; Lu, M.-C.; Chen, Z. -Y.; Yuan, Z. -W.; Xu, X. -L.; Guo, X. -K.; Zhang, X. -J.; Sun, H. -P.; You, Q. -D. J Med Chem 2015, 58, 6410.

18. Bertrand, H. C.; Schaap, M.; Baird, L.; Georgakopoulos, N. D.; Fowkes, A.; Thiollier, C.; Kachi, H.; Dinkova-Kostova, A. T.; Wells, G. J. Med. Chem. 2015, 58, 7186.

19. Lu, M. -C.; Jiao, Q.; Liu, T.; Tan, S.-J.; Zhou, H.-S; You, Q.-D.; Jiang, Z.-Y. Eur. J. Med. Chem. 2018, 143, 1578.

20. Lu, M. -C.; Chen, Z.-Y.; Wang, Y. -L.; Jiang, Y.-L.; Yuan, Z.W.; You, Q.-D.; Jiang, Z. -Y. RSC Adv. 2015, 5, 85983.

21. Spokoyny, A. M.; Zou, Y.; Ling, J. J.; Yu, H.; Lin, Y.-S, Pentelute, B. L. J. Am. Chem. Soc. 2013, 135, 5946.

22. Fadzen, C. M.; Wolfe, J. M.; Cho, C.-F.; Chiocca, E. A.; Lawler, S. E.; Pentelute, B. L. J. Am. Chem. Soc. 2017, 139, 15628.

23. Rink, H. Tetrahedron Lett. 1987,

24. For a similar assay see Shaap, M.; Hancock, R.; Wilderspin, A.; Wells, G. Protein Sci. 2013, 22, 1812.
25. Bernal, F.; Tyler, A. F.; Korsmeyer, S. J.; Walensky, L. D.; Verdine, G. L. J. Am. Chem. Soc. 2007, 129, 2456.

26. Bautista, A. D.;Appelbaum, J. S.; Craig, C. J.; Michel, J.; Schepartz, A. J. Am. Chem. Soc. 2010, 132, 2904 